\title{
The risk of low birth weight and associated factors in West Bengal, India: a community based cross-sectional study
}

\author{
Amitava Pal ${ }^{1,2^{*}}$, Sourav Manna ${ }^{3}$, Balaram Das ${ }^{4}$ and Prakash C. Dhara ${ }^{5}$
}

\begin{abstract}
Background: Low birth weight (LBW) is a challenging multifaceted public health problem as it associated with infant mortality. Present study was undertaken to assess magnitude and risk factors associated with LBW in West Bengal, India. A community-based cross-sectional study was carried out among 2611 birth episodes in different districts of West Bengal using multi-stage stratified cluster sampling, followed by systematic random sampling methodology. Participants were interviewed by semi-structured questionnaires containing socio-demographic, pregnancy, and mothers' health-related variables.
\end{abstract}

Results: This study revealed that $21.49 \%$ infants were born LBW. The risk of LBW was higher among women those age $<20$ years and $\mathrm{BMI}<18.5 \mathrm{~kg} / \mathrm{m}^{2}$. The odd of giving LBW baby were higher among women having weight $<$ $45 \mathrm{~kg}$ and height $<150 \mathrm{~cm}$. Anemic (AOR 3.33) and hypertensive (AOR 1.69) women were more likely to give LBW baby. Preterm delivery (AOR 1.93) and history of chronic illness (AOR 2.09) were the determinants of LBW. The odd of LBW was higher among women who never followed ANC visit (AOR 1.35). The likelihood of giving LBW baby was higher among women who did not consume iron folic acid tablet and additional diet during pregnancy. More LBWs were observed among women who were from low socioeconomic family, rural residence, and illiterate.

Conclusions: This study provided scientific recommendations during preconception period which will help to target women who may potentially benefit from lifestyle and dietary interventions. To reduce LBW community specific strategies such as improving awareness of the community and utilization of existing maternal health services is essential.

Keywords: Low birth weight, Maternal risk factors, Environmental risk factors, Socio economic risk factors, Prematurity

\section{Background}

Low birth weight (LBW) (birth weight $<2.5 \mathrm{~kg}$ ) is a major challenging public health problem because it is a leading cause of neonatal death and major risk factor for infant and under-five morbidity and mortality $[1,2]$. The magnitude of LBW varies from country to country.

\footnotetext{
* Correspondence: amitavaergo@gmail.com

'Deparment of Physiology, City College, 102/1, Raja Rammohan Sarani,

Kolkata, West Bengal -700009, India

2Department of Physiology, Panskura Banamali College, Purba Midnapore,

West Bengal, India

Full list of author information is available at the end of the article
}

Worldwide, out of every seven infants, one is born with LBW [3]. Globally, more than 20 million infants $(15.5 \%$ of all live births) per year are born with LBW [4]. More than 95\% of LBW infants are born in developing countries [4-6]. The magnitudes of LBW in developing countries (16.5\%) tended to be double than that of developed countries (7.0\%) [4]. In India, 30-35\% babies are LBW, however, more than half of these infants are full term babies [7]. India alone accounts for $40 \%$ of low weight babies in the overall developing countries and more than half of those born in Asia [8]. 
Proportion of babies with LBW is considered as a sensitive index of nation's health and development [9]. LBW babies have increased risk of dying in the first few weeks of life. LBW babies have a 20 times greater chance of dying in the neonatal period [5, 6]. Worldwide, 40$60 \%$ of newborn mortality is due to LBW [10]. It limits their growth and development in the child and adulthood. LBW is associated with increased risk of developing behavioral disorders, psychological disorders, and learning and sensory disabilities that impaired cognitive function of developing children and adolescents which present major challenges to them in terms of their education and quality of life $[11,12]$. LBW is associated with development of cardiovascular disease, childhood hypertension, metabolic syndrome, and diabetes in adulthood [13, 14]. LBW is also associated with increased risk abnormal neurological signs in tone and coordination and reflexes which lead to impaired motor development [15].

The causes of LBW are numerous and multifaceted. It depends on complex interactions of numerous factors like genetic, reproductive, socio-demographic, cultural, political, and surrounding physical environmental conditions and regional factors. The etiology of LBW is maximally related to maternal [16-20] and socioeconomic and psychological factors [21-23].

In spite of impressive progress in medical science and having several comprehensive programs, magnitude of LBW has little changed in India due to failure to tackle the root of LBW. Therefore, determining the magnitude and identifying the risk factors for LBW have the potential role in formulating strategies for reducing LBW in this country. The aim of the present study was to assess magnitude and risk factors associated with LBW in West Bengal, India.

\section{Methods}

\section{Study design}

The cross-sectional study design was used to describe the magnitude and associated factors of LBW.

\section{Study area and period}

The study was carried out during September 2016 to June 2018, in different districts of West Bengal state, India. West Bengal is located between $85^{\circ} 50^{\prime}$ and $89^{\circ}$ $50^{\prime}$ east longitudes and $21^{\circ} 38^{\prime}$ and $27^{\circ} 10^{\prime}$ north latitudes. The state capital is the metropolitan city of Kolkata (formerly Calcutta). As per the report of the 2011 national census, West Bengal is the fourth most crowded state in India with a population more than 91 million [24]. This contributes to $7.55 \%$ of the country's total population. The population density in this state is 1030 persons per square kilometer. The total population growth in this decade was $13.84 \%$.

\section{Sample size and sampling technique}

The required sample size was determined using single population proportion with the following assumption: $22 \%$ prevalence of LBW in West Bengal according to National Family Health Survey-III (NFHS-III) [25], 99\% confidence interval, and 3\% marginal error. As a multistage, sampling technique was employed to select respondent, a design effect of 2 was used. Also, 20\% was added for exclusions and another 20\% was added for non-responses. Thus, the final sample size was 3554 (rounded off to 3600). From an original sample of 3600 pregnant women who were registered in National Rural Health Mission, 2611 were participated in the present study. The authors disqualified 989 respondents due to multiple reasons such as migration to another geographical area (342), home delivery (251), miscarriage (49), gestational age higher than 12 weeks at the first interview (212), unknown last menstrual period (119), and twin delivery (16). Women suffering from surgical condition were not included in the study.

In the present study a multi-stage stratified cluster sampling, followed by a systematic random sampling method were employed for selecting participants from three districts (Howrah, Purba Medinipur, and Paschim Medinipur) of West Bengal. Three blocks were selected randomly from each district followed by selection of eight clusters (community health center) (six from village and two from town/municipality approximately wherever possible) from each selected block. Then systematic random sampling method was employed to select the participants from the clusters.

Before start of the present study, the study team made a short visit to the study communities to meet local community leaders, head of the family, and to the pregnant women and brief them the purpose of the study, procedures of data collection, importance of this study, and risks associated with the study. The participants agreeing to take part in the study, written consent was obtained from them during household visits. Before commencement of this study, ethical clearance and prior permission was obtained from the Institutional Ethics Review Committee.

\section{Study variables}

The main outcome variable of the study was birth weight of infants. The independent variables include socio-demographic variables (residence, religion, cast, level of education, occupation, economic condition, and socioeconomic status of household, etc.), variables related to the maternal condition (mother age, mother weight and height, BMI, hemoglobin level, blood pressure, gestational period, birth interval, number of children, chronic illness of the mother, dietary counseling during pregnancy, addition habit, maternal antenatal 
care (ANC) follow-up, iron folic acid tablet consumption, etc.), and environmental health condition (availability of latrine, type of family, and number of individuals in the house).

\section{Data collection procedure}

Pregnant women were followed monthly. A predesigned and pre-tested semi-structured questionnaire which was prepared by reviewing similar literatures was administered by house-to-house visit to the participants by the interviewers. The questionnaire included three sections. The first section of the questionnaire was related to socio-demographic background of the study participants. Second part of questionnaire contained questions related to the maternal condition, and third part of questionnaire was which help to assess household environmental conditions. The questionnaires were initially prepared in English and then translated in to Bengali. The Bengali version was again translated back to English. Prior to the actual data collection, the questionnaire was pretested on 50 pregnant women and that were not included in the main study.

The weight of the pregnant women was recorded by a portable weighing machine to the nearest $0.1 \mathrm{~kg}$ and height was recorded by anthropometer to the nearest $0.1 \mathrm{~cm}$ at the first interview at 8-12 weeks of gestation (considering negligible weight gain till 12 weeks of gestation). Hemoglobin of the respondent was measured with a finger prick sample of capillary blood and analyzed immediately using a hemoglobinometer (STAT-Site M Hemoglobin Analyzer, USA). Blood pressure was measured by auscultatory method, with the help of a sphygmomanometer (mercury type) and a stethoscope. Socioeconomic status was assessed by modified Kuppuswamy's scale [26]. All babies were weighed using a digital non-hanging type salter scale and rounded to the nearest $10 \mathrm{~g}$ within 1 day of after childbirths.

\section{Quality control of the study}

To assure the quality of the data, properly designed data collection instrument and training of data collectors were done. The data were collected by five groups of examiners (15 male and 15 female field examiners; B.Sc. in Human Physiology, fluent in the local language (Bengali)) which consist of six individuals in each group. The principle investigators and the supervisor purposively selected the members of each group. The principle investigators and the supervisor were given 2 days training to the examiners on procedures, techniques, and collecting the data including measuring weight and height, calculating gestational age, etc. While collecting data, the instruments were regularly checked for their accuracy. The principal investigators go to field to check the interview and anthropometric measurement of the field examiners and supervised and reviewed every questionnaire for completeness and logical consistency and made corrections on the spot.

\section{Statistical analysis}

Frequencies and percentages were used for categorical variables to summarize data. Difference was assessed by employing Chi-square test for categorical variables. In order to investigate the association of predictor variables with outcome variable (LBW), both bivariate and multivariate analysis were used. To determine the effect of each variable on LBW, odd ratio was calculated based on $95 \%$ confidence interval in the adjusted and unadjusted model. All analyses were performed using the Statistical Package for Social Sciences (Version 20). A $p$ value $<0.05$ was considered statistically significant.

\section{Results}

The participants tended to be young and lean; the mean age of the respondent was $22.29 \pm 3.77$ years and the mean BMI was $20.49 \pm 3.66 \mathrm{~kg} / \mathrm{m}^{2}$. Out of the respondents, majority of women (94.26\%) were below 30 years of age. About $30 \%$ of the women were underweight $\left(\mathrm{BMI}<18.5 \mathrm{~kg} / \mathrm{m}^{2}\right)$ and $10.76 \%$ were overweight/obese $\left(\right.$ BMI $\left.>25 \mathrm{~kg} / \mathrm{m}^{2}\right)$ (Table 1$)$.

More than $90 \%$ of respondent were Hindu in religion (Table 1). About $18 \%$ study participants belong to socioeconomically vulnerable groups. Majority of women belonged to lower socioeconomic classes (83.53\%) and rural residence $(82.5 \%)$. About $12 \%$ of the fathers and $10 \%$ of the mothers had no formal schooling. Most of the women were housewife (85.33\%). Large proportions of the household (13\%) in the study areas have no latrine at home and disposed excrement in the open surrounding the home. The average number of persons in a family was 5.12 people per household. The per capita income per month of the study participants was about Rs.1805/(25.42\$). More than $88 \%$ of household had a monthly per capita income less than Rs. 3000.

Out of the respondents, about 54\% women had one child (primiparous), $37.49 \%$ had two children (pauciparous), and $8.49 \%$ had more than two children (multiparous) (Table 1). Large proportion of women (38\%) had body weight less than $45 \mathrm{~kg}$. Majority of participants had a height of $150 \mathrm{~cm}$ or more (70.51\%). Out of 2611 respondents, 1658 (63.5\%) participants had their hemoglobin level less than $12 \mathrm{~g} / \mathrm{dl}$. Large percentage of the respondents was hypotensive (36.27\%). However, the prevalence of hypertension was low among them (6.89\%). History of chronic medical illness and illness during current pregnancy were noted in $5.52 \%$ and $6.51 \%$ of the study population. Engaging in hard physical work during pregnancy was fairly low (11.68\%). Large proportion of the study participants (15.2\%) did not 
Table 1 Socio-demographic and obstetric health characteristics of participants $(n=2611)$

\begin{tabular}{|c|c|c|c|c|}
\hline Variables & Category & $N(\%)$ & LBW, $n(\%)$ & $x^{2}(p)$ \\
\hline \multirow[t]{3}{*}{ Mother age (years) } & $<20$ & 736 (28.19) & $192(26.09)$ & $12.873(p<0.01)$ \\
\hline & 2029 & $1725(66.07)$ & $340(19.71)$ & \\
\hline & $\geq 30$ & $150(5.74)$ & 29 (19.33) & \\
\hline \multirow[t]{2}{*}{ Mother height (cm) } & $<150$ & 770 (29.49) & $195(25.32)$ & $9.539(p<0.01)$ \\
\hline & $\geq 150$ & $1841(70.51)$ & $366(19.88)$ & \\
\hline \multirow[t]{2}{*}{ Mother weight (kg) } & $<45$ & $986(37.76)$ & $300(30.43)$ & $75.058(p<0.001)$ \\
\hline & $\geq 45$ & $1625(62.24)$ & $261(16.06)$ & \\
\hline \multirow[t]{3}{*}{ Mother BMI $\left(\mathrm{kg} / \mathrm{m}^{2}\right)$} & $<18.5$ & $782(29.95)$ & $224(28.64)$ & $42.697(p<0.001)$ \\
\hline & $18.5-24.99$ & $1548(59.29)$ & $304(19.64)$ & \\
\hline & $>25$ & $281(10.76)$ & $33(11.74)$ & \\
\hline \multirow[t]{2}{*}{ Hemoglobin level (g/dl) } & $<12$ & $1658(63.5)$ & $452(27.26)$ & $89.828(p<0.001)$ \\
\hline & $\geq 12$ & $953(36.5)$ & 109 (11.44) & \\
\hline \multirow[t]{3}{*}{ Mother BP } & Hypotension & 947 (36.27) & $209(22.07)$ & $13.523(p<0.01)$ \\
\hline & Normotension & $1484(56.84)$ & $295(19.88)$ & \\
\hline & Hypertension & $180(6.89)$ & $57(31.67)$ & \\
\hline \multirow[t]{2}{*}{ Gestational period } & $<37$ weeks & 391 (14.98) & $132(33.76)$ & $41.065(p<0.001)$ \\
\hline & $\geq 37$ weeks & $2220(85.02)$ & $429(19.32)$ & \\
\hline \multirow[t]{3}{*}{ Parity } & Primiparous & $1536(58.83)$ & $353(22.98)$ & $10.623(p<0.01)$ \\
\hline & Pauciparous & 885 (33.9) & $159(17.97)$ & \\
\hline & Multiparous & $190(7.28)$ & $49(25.79)$ & \\
\hline \multirow[t]{2}{*}{ Inter-pregnancy interval } & $<2$ year & $369(14.13)$ & $85(23.04)$ & $0.611(p>0.05)$ \\
\hline & $>2$ year & $2242(85.87)$ & $476(21.23)$ & \\
\hline \multirow[t]{2}{*}{ History of chronic medical illness } & Yes & $144(5.52)$ & $55(38.19)$ & $25.221(p<0.001)$ \\
\hline & No & $2467(94.48)$ & $506(20.51)$ & \\
\hline \multirow[t]{2}{*}{ Illness during current pregnancy } & Yes & $170(6.51)$ & $47(27.65)$ & $4.092(p<0.05)$ \\
\hline & No & $2441(93.49)$ & $514(21.06)$ & \\
\hline \multirow[t]{2}{*}{ Dietary counseling during pregnancy } & Yes & $1953(74.8)$ & $398(20.38)$ & $5.631(p<0.05)$ \\
\hline & No & $658(25.2)$ & $163(24.77)$ & \\
\hline \multirow[t]{2}{*}{ Additional diet during pregnancy } & Yes & $1683(64.46)$ & $333(19.79)$ & $8.111(p<0.01)$ \\
\hline & No & $928(35.54)$ & $228(24.57)$ & \\
\hline \multirow[t]{2}{*}{ Hard physical work during pregnancy } & Yes & $305(11.68)$ & $91(29.84)$ & $14.273(p<0.001)$ \\
\hline & No & $2306(88.32)$ & $470(20.38)$ & \\
\hline \multirow[t]{2}{*}{ Addition habit } & Yes & $67(2.57)$ & $16(23.88)$ & $0.234(p>0.05)$ \\
\hline & No & $2544(97.43)$ & $545(21.42)$ & \\
\hline \multirow[t]{2}{*}{ Alcohol intake during pregnancy } & Yes & $34(1.3)$ & $8(23.53)$ & $0.085(p>0.05)$ \\
\hline & No & $2577(98.7)$ & $553(21.46)$ & \\
\hline \multirow[t]{2}{*}{ Fertility desire } & Yes & $275(10.53)$ & $61(22.18)$ & $0.088(p>0.05)$ \\
\hline & No & $2336(89.47)$ & $500(21.4)$ & \\
\hline \multirow[t]{2}{*}{ Iron folic acid tablet consumption } & No & $397(15.2)$ & $127(31.99)$ & $30.621(p<0.001)$ \\
\hline & Yes & $2214(84.8)$ & $434(19.6)$ & \\
\hline \multirow[t]{2}{*}{ No. of iron folic acid tablet consumption } & $<100$ & $1239(47.45)$ & $334(26.96)$ & $41.84(p<0.001)$ \\
\hline & $\geq 100$ & $1372(52.55)$ & $227(16.55)$ & \\
\hline \multirow[t]{2}{*}{ ANC follow-up status } & No & $180(6.89)$ & $50(27.78)$ & $4.537(p<0.05)$ \\
\hline & Yes & $2431(93.11)$ & $511(21.02)$ & \\
\hline
\end{tabular}


Table 1 Socio-demographic and obstetric health characteristics of participants $(n=2611)$ (Continued)

\begin{tabular}{|c|c|c|c|c|}
\hline Variables & Category & $N(\%)$ & LBW, $n(\%)$ & $x^{2}(p)$ \\
\hline \multirow[t]{2}{*}{ No. of ANC visit } & $<3$ & $597(22.86)$ & $154(25.8)$ & $8.521(p<0.01)$ \\
\hline & $\geq 3$ & $2014(77.14)$ & $407(20.21)$ & \\
\hline \multirow[t]{3}{*}{ Time of 1st ANC visit } & 1st trimester & $1684(64.5)$ & 345 (20.49) & $2.948(p>0.05)$ \\
\hline & 2nd trimester & 851 (32.59) & $197(23.15)$ & \\
\hline & 3rd trimester & $76(2.91)$ & $19(25)$ & \\
\hline \multirow[t]{2}{*}{ Child sex } & Male & $1330(50.9)$ & $275(20.68)$ & $1.053(p>0.05)$ \\
\hline & Female & $1281(49.1)$ & $286(22.33)$ & \\
\hline \multirow[t]{2}{*}{ Residence } & Rural & $2154(82.5)$ & $488(22.66)$ & $9.978(p<0.01)$ \\
\hline & Urban & $457(17.5)$ & $73(15.97)$ & \\
\hline \multirow[t]{2}{*}{ Religion } & Hindu & $2357(90.27)$ & $513(21.76)$ & $1.117(p>0.05)$ \\
\hline & Muslim & $254(9.73)$ & $48(18.9)$ & \\
\hline \multirow[t]{2}{*}{ Cast } & General & $2129(81.54)$ & $416(19.54)$ & $25.9(p<0.001)$ \\
\hline & SC/ST & $482(18.46)$ & $145(30.08)$ & \\
\hline \multirow[t]{3}{*}{ Educational status of father } & Illiterate/ able to sign & $318(12.18)$ & $100(31.45)$ & $21.338(p<0.001)$ \\
\hline & Primary & $1129(43.24)$ & $229(20.28)$ & \\
\hline & Secondary or above & $1164(44.58)$ & $232(19.93)$ & \\
\hline \multirow[t]{3}{*}{ Educational status of mother } & Illiterate/ able to sign & $272(10.42)$ & $80(29.41)$ & $13.399(p<0.01)$ \\
\hline & Primary & $1297(49.67)$ & $281(21.67)$ & \\
\hline & Secondary or above & $1042(39.91)$ & $200(19.19)$ & \\
\hline \multirow[t]{2}{*}{ Father occupation } & Laborers/cultivator & $1722(65.95)$ & 406 (23.58) & $13.111(p<0.001)$ \\
\hline & Business/service & 889 (34.05) & $155(17.44)$ & \\
\hline \multirow[t]{3}{*}{ Mother occupation } & Cultivator & $262(10.03)$ & 81 (30.92) & $15.505(p<0.001)$ \\
\hline & Housewife & $2228(85.33)$ & $457(20.51)$ & \\
\hline & Service & $121(4.63)$ & $23(19.01)$ & \\
\hline \multirow[t]{3}{*}{ Housing condition } & Kanch & 996 (38.15) & $232(23.29)$ & $3.122(p>0.05)$ \\
\hline & Semi pacca & $631(24.17)$ & $128(20.29)$ & \\
\hline & Pacca & $984(37.69)$ & $201(20.43)$ & \\
\hline \multirow[t]{2}{*}{ Availability of latrine } & No & $350(13.4)$ & $108(30.86)$ & $21.04(p<0.001)$ \\
\hline & Yes & 2261 (86.6) & $453(20.04)$ & \\
\hline \multirow[t]{3}{*}{ Income (Rs) } & $<1000$ & $673(25.78)$ & $171(25.41)$ & $10.116(p<0.01)$ \\
\hline & $1000-3000$ & $1630(62.43)$ & 337 (20.67) & \\
\hline & $>3000$ & $308(11.8)$ & $53(017.21$ & \\
\hline \multirow[t]{2}{*}{ Family size } & $\leq 4$ & $1172(44.89)$ & $263(22.44)$ & $1.148(p>0.05)$ \\
\hline & $>4$ & $1439(55.11)$ & $298(20.71)$ & \\
\hline \multirow[t]{3}{*}{ Socioeconomic status } & Lower/upper lower & $1241(47.53)$ & 294 (23.69) & $15.509(p<0.001)$ \\
\hline & Middle/lower middle & $940(36)$ & $204(21.7)$ & \\
\hline & Upper/upper middle & $430(16.47)$ & 63 (14.65) & \\
\hline
\end{tabular}

consume iron folic acid tablets during current pregnancy or consume less than recommended (47.45\%) (100 iron folic acid tablet). Similarly, large proportion of the study participants $(35.54 \%)$ did not consume additional diet during current pregnancy. Alcohol intake during pregnancy was fairly low (1.3\%). Majority of women (93.11\%) had followed ANC visit; however, about 23\% had less than three follow-up during current pregnancy.

The mean birth weight and gestational age of infants were $2.74 \mathrm{~kg}$ and 39.65 week. A total of 561 (21.49\%) were classified as LBW, and 391 infants (14.98\%) were born preterm. Among the total respondents, 83 (3.18\%) 
infants were born with birth weight less than $2 \mathrm{~kg}$, and 33 (1.26\%) infants were born before 32 weeks of gestation. Out of 391 preterm infants, 132 (33.76\%) infants were born with birth weight less than $2.5 \mathrm{~kg}$.

\section{Determinants of LBW}

Bivariate logistic regression analyses were performed to study the association between different risk factors and the occurrence of LBW and have been summarized in Table 2. The analyses revealed that preterm delivery, maternal age, height and weight of mother, maternal BMI, hemoglobin level, blood pressure, parity, history of chronic illness, illness during current pregnancy, dietary counseling and additional diet during pregnancy, hard physical work, iron folic acid tablet consumption, ANC follow-up, residence, cast, educational and occupational status of parents, availability of latrine, per capita income, and socioeconomic status were significantly associated with LBW.

Those variables which have significant association with LBW in the bivariate model were subjected to the multivariate analysis. The risk of LBW tended to be 1.21 times higher among the women whose ages less than 20 years than those ages between 20 and 30 years. The risk of LBW tended to be 1.12 times among women with BMI less than $18.5 \mathrm{~kg} / \mathrm{m}^{2}$ as compared to normal-weight women (BMI, $18.5-24.99 \mathrm{~kg} / \mathrm{m}^{2}$ ); however, the risk of LBW was lower (AOR, 0.62; 95\% CI, 0.41-0.95) among women whose BMI was $25 \mathrm{~kg} / \mathrm{m}^{2}$ or more. The odd of giving LBW baby was 1.7 times higher among women whose weight less than $45 \mathrm{~kg}$. Maternal short height (< $150 \mathrm{~cm}$ ) was a risk factor (AOR, 1.28; 95\% CI, 1-1.65) for LBW. Anemic (AOR, 3.33; 95\% CI, 2.59-4.28) and hypertensive (AOR, 1.69; 95\% CI, 1.09-2.61) women were more likely to give LBW baby. Preterm delivery (AOR, 1.93; 95\% CI, 1.49-2.5) and history of chronic medical illness (AOR, 2.09; 95\% CI, 1.38-3.15) were determinants of LBW. The odd of LBW was higher among the participants who never followed ANC visit (AOR, 1.35; 95\% CI, 0.99-1.74). More LBW babies were observed among women who attended ANC less than three times (AOR, 1.4; 95\% CI, 1.04-1.87) in current pregnancy as compared to women who attended ANC three times or more. The likelihood of giving LBW baby was significantly higher among women who did not consume an additional diet during pregnancy (AOR, 1.45; 95\% CI, 1.03-2.03). In the same manner, women who did not consume iron folic acid tablets during current pregnancy were about four times (AOR, 3.86; 95\% CI, 2.54-5.87) more prone to deliver LBW babies. Women who take iron folic acid tablets partially $(<100$ tablets $)$ were more likely to give LBW babies (AOR, 1.48; 95\% CI, 1.18-1.87) than those who took a full course. More LBWs were observed among women who are residing in rural areas and from low socioeconomic families than their counterparts. Compared to parity, multiparous women were more likely to deliver LBW babies than those of primiparous women.

\section{Discussion}

In the last three to two decades, there has been profound economic development in India [27]. As per the Central Statistics Organization and International Monetary Fund, India has emerged as the fastestgrowing economy in the world. However, India still spends only around $4.7 \%$ of its GDP toward healthcare compared to the USA (18\%). As per the World Health Report ranking, India's healthcare system is at 112 out of 190 countries [28]. India did not achieve the fourth Millennium Development Goals (MDG) to reduce infant and child mortality by two-thirds between 1990 and 2015. LBW is one of the major causes of infant and child mortality in India.

This study presented the report of 2611 birth episodes from different districts of West Bengal, India. This study revealed that nearly one-fifth $(21.49 \%)$ infants were LBW. As per NFHS-III, prevalence of LBW in West Bengal was 22\% [25]. Present study depicted actual scenario that the prevalence of LBW has not been significantly reduced over 10 years. The magnitude of LBW in different regions of India was reported by several researchers. The prevalence of LBW was higher in a community-based study in Bhopal of which about $43.6 \%$ [29]. The incidence of LBW was $23.9 \%$ in Tripura, the eastern state of India [30]. In another study from Tamil Nadu conducted by Dandekar et al. [31] reported that the frequency rate of LBW was $11.67 \%$. The prevalence of LBW was higher in Northern India (32.3\%) than that of the present study [21]. Comparison with global estimates, the prevalence of LBW in this study area was higher than those reported in Sri Lanka (8.7\%) [32], Malaysia (12.6\%) [33], Tanzania (13.6\%) [22], Iran (8.8\%) [20], and Ethiopia (14.6\%) [6], but lower than that of Nepal (34.37\%) [34].

In the present study, several maternal and sociodemographic characteristics were associated with LBW. This study showed that teenage mothers had delivered significantly more LBW babies. Similar to our study, different other studies also showed similar findings [35]. NFHS-III in India also confirmed that the proportion of LBW was lesser among older women [25]. Dasgupta and Basu [19] reported that the mothers aged below 20 years had significantly greater chance to deliver LBW baby than the age group of above 20 years. The odd of having a LBW baby was higher among multiparous women than those of primiparous. This may be due to short interpregnancy interval $[16,20]$. 
Table 2 Logistic regression output of determinants of LBW birth

\begin{tabular}{|c|c|c|c|c|c|}
\hline Variables & Category & COR (95th Cl) & $p$ value & AOR (95th Cl) & $p$ value \\
\hline \multirow[t]{3}{*}{ Mother age (years) } & $<20$ & $1.44(1.17-1.76)$ & 0.000 & $1.21(0.94-1.54)$ & 0.138 \\
\hline & $20-29$ & Reference & & Reference & \\
\hline & $\geq 30$ & $0.98(0.64-1.49)$ & 0.911 & $1.05(0.64-1.73)$ & 0.837 \\
\hline \multirow[t]{2}{*}{ Mother height $(\mathrm{cm})$} & $<150$ & $1.37(1.12-1.67)$ & 0.002 & $1.28(1-1.65)$ & 0.05 \\
\hline & $\geq 150$ & Reference & & Reference & \\
\hline \multirow[t]{2}{*}{ Mother weight (kg) } & $<45$ & $2.28(1.89-2.76)$ & 0.000 & $1.7(1.27-2.29)$ & 0.000 \\
\hline & $\geq 45$ & Reference & & Reference & \\
\hline \multirow[t]{3}{*}{ Mother BMI (kg/m²) } & $<18.5$ & $1.64(1.35-2)$ & 0.000 & $1.12(0.82-1.52)$ & 0.485 \\
\hline & $18.5-24.99$ & Reference & & Reference & \\
\hline & $>25$ & $0.54(0.37-0.8)$ & 0.002 & $0.62(0.41-0.95)$ & 0.027 \\
\hline \multirow[t]{2}{*}{ Hemoglobin level (g/dl) } & $<12$ & $2.9(2.31-3.64)$ & 0.000 & $3.33(2.59-4.28)$ & 0.000 \\
\hline & $\geq 12$ & Reference & & Reference & \\
\hline \multirow[t]{3}{*}{ Mother BP } & Hypotension & $1.14(0.93-1.39)$ & 0.194 & $0.91(0.72-1.13)$ & 0.381 \\
\hline & Normotension & Reference & & Reference & \\
\hline & Hypertension & $1.87(1.33-2.62)$ & 0.000 & $1.69(1.09-2.61)$ & 0.019 \\
\hline \multirow[t]{2}{*}{ Gestational period } & $<37$ weeks & $2.13(1.68-2.69)$ & 0.000 & $1.93(1.49-2.5)$ & 0.000 \\
\hline & $\geq 37$ weeks & Reference & & Reference & \\
\hline \multirow[t]{3}{*}{ Parity } & Primiparous & $1.36(1.11-1.68)$ & 0.004 & $1.18(0.92-1.52)$ & 0.191 \\
\hline & Pauciparous & Reference & & Reference & \\
\hline & Multiparous & $1.59(1.1-2.29)$ & 0.014 & $2.31(1.14-3.51)$ & 0.000 \\
\hline \multirow[t]{2}{*}{ Inter-pregnancy interval } & $<2$ year & $1.11(0.85-1.44)$ & 0.434 & & \\
\hline & $>2$ year & Reference & & & \\
\hline \multirow[t]{2}{*}{ History of chronic medical illness } & Yes & $2.39(1.69-3.4)$ & 0.000 & $2.09(1.38-3.15)$ & 0.000 \\
\hline & No & Reference & & Reference & \\
\hline \multirow[t]{2}{*}{ IIIness during current pregnancy } & Yes & $1.43(1.01-2.03)$ & 0.044 & $1.34(0.89-2.02)$ & 0.16 \\
\hline & No & Reference & & Reference & \\
\hline \multirow[t]{2}{*}{ Dietary counseling during pregnancy } & Yes & Reference & & Reference & \\
\hline & No & $1.29(1.04-1.58)$ & 0.018 & $0.97(0.66-1.42)$ & 0.886 \\
\hline \multirow[t]{2}{*}{ Additional diet during pregnancy } & Yes & Reference & & Reference & \\
\hline & No & $1.32(1.09-1.6)$ & 0.004 & $1.45(1.03-2.03)$ & 0.032 \\
\hline \multirow[t]{2}{*}{ Hard physical work during pregnancy } & Yes & $1.66(1.27-2.17)$ & 0.000 & $1.07(0.58-1.99)$ & 0.818 \\
\hline & No & Reference & & Reference & \\
\hline \multirow[t]{2}{*}{ Addition habit } & Yes & $1.15(0.65-2.03)$ & 0.629 & & \\
\hline & No & Reference & & & \\
\hline \multirow[t]{2}{*}{ Alcohol intake during pregnancy } & Yes & $1.13(0.51-2.5)$ & 0.77 & & \\
\hline & No & Reference & & & \\
\hline \multirow[t]{2}{*}{ Fertility desire } & Yes & Reference & & & \\
\hline & No & $1.05(0.77-1.41)$ & 0.766 & & \\
\hline \multirow[t]{2}{*}{ Iron folic acid tablet consumption } & No & $1.95(1.54-2.46)$ & 0.000 & $3.86(2.54-5.87)$ & 0.000 \\
\hline & Yes & Reference & & Reference & \\
\hline \multirow[t]{2}{*}{ No. of iron folic acid tablet consumption } & $<100$ & $1.86(1.54-2.25)$ & 0.000 & $1.48(1.18-1.87)$ & 0.001 \\
\hline & $\geq 100$ & Reference & & Reference & \\
\hline \multirow[t]{2}{*}{ ANC follow-up status } & No & $1.45(1.03-2.03)$ & 0.034 & $1.35(0.99-1.74)$ & 0.037 \\
\hline & Yes & Reference & & Reference & \\
\hline
\end{tabular}


Table 2 Logistic regression output of determinants of LBW birth (Continued)

\begin{tabular}{|c|c|c|c|c|c|}
\hline Variables & Category & COR (95th Cl) & $p$ value & AOR (95th Cl) & $p$ value \\
\hline \multirow[t]{2}{*}{ No. of ANC visit } & $<3$ & $1.37(1.11-1.7)$ & 0.004 & $1.4(1.04-1.87)$ & 0.025 \\
\hline & $\geq 3$ & Reference & & Reference & \\
\hline \multirow[t]{3}{*}{ Time of 1st ANC visit } & 1st trimester & Reference & & & \\
\hline & 2nd trimester & $1.17(0.96-1.43)$ & 0.123 & & \\
\hline & 3rd trimester & $1.29(0.76-2.2)$ & 0.343 & & \\
\hline \multirow[t]{2}{*}{ Child sex } & Male & Reference & & & \\
\hline & Female & $1.1(0.91-1.33)$ & 0.305 & & \\
\hline \multirow[t]{2}{*}{ Residence } & Rural & $1.54(1.18-2.02)$ & 0.002 & $2.6(1.43-4.08)$ & 0.000 \\
\hline & Urban & Reference & & Reference & \\
\hline \multirow[t]{2}{*}{ Religion } & Hindu & $1.19(0.86-1.66)$ & 0.291 & & \\
\hline & Muslim & Reference & & & \\
\hline \multirow[t]{2}{*}{ Cast } & General & Reference & & Reference & \\
\hline & SC/ST & $1.77(1.42-2.21)$ & 0.000 & $0.99(.74-1.34)$ & 0.972 \\
\hline \multirow[t]{3}{*}{ Educational status of father } & Illiterate/ able to sign & $1.84(1.4-2.43)$ & 0.000 & $1.12(0.7-1.78)$ & 0.633 \\
\hline & Primary & $1.02(0.83-1.25)$ & 0.833 & $0.81(0.61-1.08)$ & 0.155 \\
\hline & Secondary or above & Reference & & Reference & \\
\hline \multirow[t]{3}{*}{ Educational status of mother } & Illiterate/able to sign & $1.75(1.3-2.37)$ & 0.000 & $1.06(0.66-1.68)$ & 0.822 \\
\hline & Primary & $1.16(0.95-1.43)$ & 0.142 & $1.05(0.81-1.35)$ & 0.729 \\
\hline & Secondary or above & Reference & & Reference & \\
\hline \multirow[t]{2}{*}{ Father occupation } & Laborers/cultivator & $1.46(1.19-1.79)$ & 0.000 & $1.05(0.79-1.39)$ & 0.756 \\
\hline & Business/service & Reference & & Reference & \\
\hline \multirow[t]{3}{*}{ Mother occupation } & Cultivator & $1.91(1.13-3.22)$ & 0.016 & $0.89(0.41-1.94)$ & 0.763 \\
\hline & Housewife & $1.1(0.69-1.75)$ & 0.69 & $0.87(0.48-1.58)$ & 0.658 \\
\hline & Service & Reference & & Reference & \\
\hline \multirow[t]{3}{*}{ Housing condition } & Kanch & $1.18(0.95-1.46)$ & 0.123 & & \\
\hline & Semi pacca & $0.99(0.77-1.27)$ & 0.945 & & \\
\hline & Pacca & Reference & & & \\
\hline \multirow[t]{2}{*}{ Availability of latrine } & No & $1.78(1.39-2.28)$ & 0.000 & $1.18(0.84-1.66)$ & 0.33 \\
\hline & Yes & Reference & & Reference & \\
\hline \multirow[t]{3}{*}{ Income (Rs) } & $<1000$ & $1.64(1.16-2.31)$ & 0.005 & $1.12(0.71-1.77)$ & 0.63 \\
\hline & $1000-3000$ & $1.25(0.91-1.73)$ & 0.165 & $1.00(0.66-1.51)$ & 0.994 \\
\hline & $>3000$ & Reference & & Reference & \\
\hline \multirow[t]{2}{*}{ Family size } & $\leq 4$ & $1.11(0.92-1.34)$ & 0.284 & & \\
\hline & $>4$ & Reference & & & \\
\hline \multirow[t]{3}{*}{ Socioeconomic status } & Lower/upper lower & $1.81(1.34-2.43)$ & 0.000 & $1.64(0.98-2.75)$ & 0.062 \\
\hline & Middle/lower middle & $1.61(1.18-2.2)$ & 0.002 & $1.52(0.99-2.33)$ & 0.058 \\
\hline & Upper/upper middle & Reference & & Reference & \\
\hline
\end{tabular}

Our study revealed that maternal undernutrition (BMI $<18 \mathrm{~kg} / \mathrm{m}^{2}$ ) was significantly associated with LBW. This was consistent with earlier studies done in India [36] and abroad [16, 22, 37]. Wataba et al. [37] reported that an underweight woman was two times prone to deliver LBW babies than normal-weight women. The association between maternal underweight and LBW might be due to lack of nutrients and or medical illness of the women resulting in diminished fetal growth [16]. The risk having LBW baby was $70 \%$ higher among women whose weight is less than $45 \mathrm{~kg}$ and $28 \%$ higher whose height is less than $150 \mathrm{~cm}$. This finding is in line with several studies [18, 36, 38]. Maternal height and weight might affect intrauterine growth of the fetus [7, 38]. 
This study has shown that maternal anemia and hypertension were significantly associated with LBW. Similar finding was also reported by several researchers [17, 18, 23]. Anemia could impair oxygen delivery to the fetus and thus interfere with normal intrauterine growth [38]. Maternal hypertension is thought to cause of LBW by affecting placental blood flow thus limiting nutrient supply [17]. The odd of having LBW baby was higher among women with history of chronic medical illness. This finding was in agreement with several studies [6, 38]. A stronger association between prematurity and LBW were pointed in this study. The association has already been described worldwide, and it has been considered the main factor for keeping LBW [6, 22].

This study showed that women who resided in rural areas and from lower socioeconomic families were more prone to deliver LBW babies. This result was consistent with earlier studies [16, 22]. The difference might be due to inadequate health information, inaccessibility of medical services, and lack of nutritional awareness among rural women. Rural women from lower socioeconomic families are more prone to poor diet and infection and more likely to undertake physically demanding work [23, 39]. Additionally, there are wide gaps in the healthcare system in India between the rural and urban areas. The risk of LBW was higher among women who never followed ANC visit or attend ANC less than three times. This finding was consistent with an earlier study done in Nepal [10]. Utilization of ANC services is an established factor to improve pregnancy outcomes and to reducing maternal mortality. In India, through ANC service appropriate nutritional education, iron supplementation along with folic acid, tetanus toxoid vaccine, timely health check-up, monitoring the fetal well-being, etc are provided to the pregnant women to improve pregnancy outcome. However, large proportion of pregnant women from the rural area of India are not utilizing or inadequately utilizing antenatal care services due to lack of awareness. Participants who did not consume iron folic acid tablets or consumed partially $(<100$ tablets) during the current pregnancy were more likely to give LBW babies. Our study was consistent with a study conducted in Tripura, India [30]. Khanal et al. [40] reported that iron supplementation during pregnancy can prevent LBW.

Parental education was significantly associated with LBW in the bivariate model. Similar to other studies, this study was also found more proportion of LBW among illiterate or less educated parents [16, 21]. Education plays an important role in improving health-seeking behavior, social status, and living standard and health awareness such as proper maternal feeding practices, maternal health service utilization, etc $[16,21]$. This study revealed that women who were in lower-income level were at higher risk to deliver LBW babies. Similarly, the study conducted in Ethiopia showed that per capita income of the family was significantly associated with the birth weight of the newborn [16]. The household environmental factor such as latrine availability was statistically associated with LBW in the bivariate analysis. Open defecation and unsafe excreta disposal continue to be negatively affecting health and nutrition status of pregnant women and promotion of chronic diseases [25].

\section{Conclusion}

The findings of this study showed that various sociodemographic, maternal, and household environmental variables were risk factors for LBW. Illiteracy, rural residence, poor economy, low socioeconomic status, and poor sanitation were identified as risk factors for LBW. Absence in ANC follow-up, teenage pregnancy, maternal low weight, and short height, not consuming additional diet and iron folic acid tablet, and multiparity were identified as risk factors for LBW. Maternal undernutrition, anemia, hypertension, and history of chronic medical illness have increased the risk of LBW baby. Therefore, prevention strategy should be designed to tackle these multiple risk factors for LBW in this area. Through ANC, it is possible to identity a woman who is at risk of having a LBW baby. Followers of ANC should receive disease-specific counseling by skilled health personnel with emphasis given to women with chronic medical illnesses. Giving necessary suggestions such as the need for additional balance diet and provision of iron folic acid tablets for the anemic can be easily facilitated. These activities would not only prevent occurrence of LBW but also prevent problems associated with LBW such as child morbidity and mortality, thereby helping the country to achieve MDG targets.

\section{Study limitation}

Despite efficient sample design, large sample size, and robust analyses, this study is not free from certain limitations. The findings of this study might be influenced by purposive selection of study area and study design bias. Other limitations are possible recall bias while determining the gestational age and inability to include women who deliver at home. There may be desirability bias for monthly income and socio-demographic variables. Thus, this may overestimate the association between the predictors and the outcome variable. However, we reduce this probably bias with attention to parent's occupation. In the present study, different predictors for LBW were studied, however, some important other potential risk factors for LBW including maternal 
psychological stress, domestic violence, toxic exposures, and quality of antenatal care received by the pregnant women, which may have some effect on LBW were not studied. In the present study, the participants were selected from different districts of West Bengal, India. Therefore, the generalizability of findings of this study is not applicable for all Indian participants.

\section{Abbreviations}

LBW: Low birth weight; ANC: Antenatal care; AOR: Adjusted odd ratio; COR: Crude odd ratio; MDG: Millennium development goals; NFHSIII: National Family Health Survey-III

\section{Acknowledgements}

All the authors would like to express their gratitude and thanks to the study participants and data collectors for their great contribution for the completion of this study.

\section{Authors' contributions}

Conceived and designed experiments: Amitava Pal and Prakash C. Dhara. Analyzed the data: Amitava Pal, Sourav Manna, and Balaram Das. Wrote the paper: Amitava Pal. Carried out literature review: Amitava Pal, Sourav Manna, and Balaram Das. Reviewed and approved the final version of the manuscript: Amitava Pal, Sourav Manna, Balaram Das, and Prakash C. Dhara.

\section{Funding}

The study does not have a funding source.

\section{Availability of data and materials}

The datasets used and/or analyzed during the current study are available from the corresponding author on reasonable request.

\section{Ethics approval and consent to participate}

Before the commencement of this study, ethical clearance and prior permission were obtained from the Institutional Ethics Review Committee (Ethical No.: VU/R/Ethical/3-(ii)/2012; dated: 22 March 2012). The participants agreeing to take part in the study, written consent was obtained from them during household visits.

\section{Consent for publication}

Not applicable

\section{Competing interests}

The authors declare that they have no competing interests.

\section{Author details}

'Deparment of Physiology, City College, 102/1, Raja Rammohan Sarani, Kolkata, West Bengal -700009, India. ${ }^{2}$ Department of Physiology, Panskura Banamali College, Purba Midnapore, West Bengal, India. ${ }^{3}$ Department of Physiology, National Medical College, Birgunj, Parsa, Nepal. ${ }^{4}$ Department of Physiology, Belda College, Paschim Midnapore, West Bengal, India. ${ }^{5}$ Ergonomics and Sports Physiology Division, Department of Human Physiology, Vidyasagar University, Midnapore, West Bengal, India.

Received: 13 February 2020 Accepted: 10 August 2020

Published online: 01 September 2020

\section{References}

1. Vermeulen GM (2000) Spontaneous preterm birth: prevention, management and outcome. Eur J Obstet Gynecol Reprod Biol 93:1-3

2. Ganchimeg T, Ota E, Morisaki N, Laopaiboon M, Lumbiganon P, Zhang J et al (2014) On behalf of the WHO multicountry survey on maternal newborn health research network. Pregnancy and childbirth outcomes among adolescent mothers: a World Health Organization multicountry study. BJOG 121(Suppl. 1):40-48

3. Kayode GA, Amoakoh-Coleman M, Agyepong IA, Ansah E, Grobbee DE, Klipstein-Grobusch K (2014) Contextual risk factors for low birth weight: a multilevel analysis. PLoS One 9(10):e109333. https://doi.org/10.1371/journal. pone.0109333
4. Feresu SA, Harlow SD, Woelk GB (2015) Risk factors for low birth weight in Zimbabwean women: a secondary data analysis. PLoS One 10(6):e0129705. https://doi.org/10.1371/journal.pone.0129705

5. United Nations Children's Fund and WHO (2004) Low birth weight country, regional and global estimates, New York http://www.unicef.org/ publications/index_24840.html

6. Gebremedhin M, Ambaw F, Admassu E, Berhane H (2014) Maternal associated factors of low birth weight: a hospital based cross-sectional mixed study in Tigray, Northern Ethiopia. BMC Pregnancy Childbirth 15:222. https://doi.org/10.1186/s12884-015-0658-1

7. Dalal A, Chauhan S, Bala DV (2014) Epidemiological determinants of low birth weight in Ahmedabad city: a facility-based case-control study. Int J Med Sci Public Health 3:430-432

8. Reddy RS, Sarma W (2015) Comparative study of socio-economic status of mothers who delivered term low birth weight babies with mothers who delivered normal birth weight babies in a tertiary care rural hospital. Int Archives of Integrated Med 2(5):129-134

9. Idris MZ, Gupta A, Mohan U, Srivastava AK, Das V (2000) Maternal health and low birth weight among institutional deliveries. Indian J Community Med 25(4):156-160

10. Sharma SR, Giri S, Timalsina U, Bhandari SS, Basyal B, Wagle K et al (2015) Low birth weight at term and its determinants in a tertiary hospital of Nepal: a case-control study. PLoS One 10(4):e0123962. https://doi.org/10. 1371/journal.pone.0123962

11. Hollo O, Rautava P, Korhonen T, Helenius H, Kero P, Sillanpapa M (2006) Academic achievement of small-for-gestational age children at age 10 years. Arch Pediatr Adolesc Med 56:179-187

12. McAvoy H, Sturley J, Burke S, Balanda K (2006) Unequal at birth: inequalities in the occurrence of low birth weight babies in Ireland. The Institute of Public Health in Ireland. Forestview, Bishop's Square Purdy's Lane, Redmond's Hill Belfast, Dublin 2 BT8 7ZX, Ireland.

13. Barker DJ, Forsén T, Uutela A, Osmond C, Eriksson JG (2001) Size at birth and resilience to effects of poor living conditions in adult life: longitudinal study. BMJ 323:1273-1276

14. Borghese B, Sibiude J, Santulli P, Lafay Pillet MC, Marcellin L, Brosens I et al (2015) Low birth weight is strongly associated with the risk of deep infiltrating endometriosis: results of a 743 case-control study. PLoS One 10(2):e0117387. https://doi.org/10.1371/journal.pone.0117387

15. Pal A, Manna S, Dhara PC (2019) Comparison between the motor function of school-aged children with normal birth weight and children with low birth weight: a cross-sectional study. Turk J Pediatr 61:374-385

16. Demelash H, Motbainor A, Nigatu D, Gashaw K, Melese A (2015) Risk factors for low birth weight in Bale zone hospitals, South-East Ethiopia: a casecontrol study. BMC Pregnancy Childbirth 15:264. https://doi.org/10.1186/ s12884-015-0677-y

17. Hossain N, Khan N, Khan NH (2009) Obstetric causes of stillbirth at low socioeconomic settings. J Pak Med Assoc 59(11):744-747

18. Sachin SM, Girish M, Rajesh D, Surekha Y, Madhav KT, Kiran P (2012) Maternal risk factors associated with term low birth weight neonates: a matched-pair case control study. Indian Pediatr 49:25-28

19. Dasgupta A, Basu R (2011) Determinants of low birth weight in a block of Hooghly, West Bengal: a multivariate analysis. Int J Biol Med Res 2(4):838-842

20. Golestan M, Akhavan Karbasi S, Fallah R (2011) Prevalence and risk factors for low birth weight in Yazd, Iran. Singapore Med J 52(10):730-733

21. Agarwal G, Ahmad S, Goel K, Kumar V, Goel P, Garg M et al (2012) Maternal risk factors associated with low birth weight neonates in a tertiary care hospital, Northern India. J Community Med Health Educ 2:177. https://doi. org/10.4172/2161-0711.1000177

22. Siza JE (2008) Risk factors associated with low birth weight of neonates among pregnant women attending a referral hospital in Northern Tanzania. Tanzan J Health Res 10(1):5-8

23. Meshram II, Rao MK, Reddy CG, Ravindranath M, Kumar SS, Sreerama KK et al (2016) Prevalence of under nutrition and its predictors among under 5 year children in Surat Region, Gujarat, India. J Clin Nutr \& Diet 2:1

24. Census of India (2011) Office of the Registrar General \& Census Commissioner, New Delhi, India. https://censusindia.gov.in/2011-common/ censusdata2011.html

25. National fact sheet, India, 2005-2006. National Family Health Survey (NFHSIII). http://www.nfhsindia.org/pdf/IN.pdf. Accessed 24 Dec 2018 
26. Kumar BPR, Dudala SR, Rao AR (2013) Kuppuswamy's socio-economic status scale - a revision of economic parameter for 2012. Int J Res Dev Health 1(1):2-4

27. Pal A, Pari AK, Sinha A, Dhara PC (2017) Prevalence of undernutrition and associated factors: a cross-sectional study among rural adolescents in West Bengal, India. International Journal of Pediatrics and Adolescent Medicine 4: 9-18

28. The World health report (2000) Health systems: improving performance. http://www.who.int/whr/2000/en/whr00_en.pdf. Accessed 16 Sept 2018

29. Choudhary AK, Choudhary A, Tiwari SC, Dwivedi R (2013) Factors associated with low birth weight among newborns in an urban slum community in Bhopal. Indian J Public Health 57:20-23

30. Bhattacharjya H, Das S, Ghosh D (2015) Proportion of low birth weight and related factors in a tertiary care institute of Tripura. International Journal of Medicine and Public Health 5:10-13

31. Dandekar RH, Shafee M, Sinha SP (2014) Prevalence and risk factors affecting low birth weight in a district hospital at Perambalur, Tamilnadu. Global J Med Publ Health 3(2):1-5

32. Jananthan R, Wijesinghe DGNG, Sivananthawerl T (2009) Maternal anthropometry as a predictor of birth weight. Trop. Agric Res 21(1):89-98

33. Boo NY, Lim SM, Koh KT, Lau KF, Ravindran J (2008) Risk factors associated with low birth weight infants in the Malaysian Population. Med J Malaysia 63(4):306-310

34. Joshi HS, Srivastava PC, Agnihotri AK, Joshi MC, Shalini C, Vipul M (2010) Risk factors for low birth weight (LBW) babies and its medico legal significance. J Indian Acad Forensic Med 32(3):212-215

35. Negi KS, Kandpal SD, Kukreti M (2006) Epidemiological factors affecting low birth weight. JK Science 8(1):31-34

36. Nagargoje M (2007) A case control study for risk factors of low birth weight in Nagpur city of Maharashtra, India. Indian J Community Health 22(2):1-4

37. Wataba K, Mizutani T, Morine M, Sugiyama T, Suehara N (2006) Impact of prepregnant body mass index and maternal weight gain on the risk of pregnancy complications in Japanese women. Acta Obstet Gynecol Scand 85(3):1-15

38. Kramer MS (1987) Determinants of low birth weight: methodological assessment and meta-analysis. Bull World Health Organ 65(5):663-737

39. Pal A, De S, Sengupta P, Maity P, Dhara PC (2014) An investigation on prevalence of anemia in relation to BMI and nutrient intake among adult rural population of West Bengal, India. Epidemiology, Biostatistics and. Public Health 11(2):e8915-1-e8915-10

40. Khanal V, Zhao Y, Sauer K (2014) Role of antenatal care and iron supplementation during pregnancy in preventing low birth weight in Nepal: comparison of national surveys 2006 and 2011. Arch Public Health 72:4. https://doi.org/10.1186/2049-3258-72-4

\section{Publisher's Note}

Springer Nature remains neutral with regard to jurisdictional claims in published maps and institutional affiliations.

\section{Submit your manuscript to a SpringerOpen ${ }^{\circ}$ journal and benefit from:}

- Convenient online submission

- Rigorous peer review

- Open access: articles freely available online

- High visibility within the field

- Retaining the copyright to your article

Submit your next manuscript at $\boldsymbol{\nabla}$ springeropen.com 